\title{
Anchored boundary conditions for locally isostatic networks
}

\author{
Louis Theran* \\ Aalto Science Institute (AScI) and Department of Computer Science (CS), \\ Aalto University, PO Box 15500, 00076 Aalto, Finland \\ Anthony Nixon ${ }^{\dagger}$ \\ Department of Mathematics and Statistics, Lancaster University, Lancaster LA1 4 YF, England \\ Elissa Ros $¥$ \\ MESH Consultants Inc., Fields Institute for Research in the Mathematical Sciences, \\ 222 College Street, Toronto, ON, M5T 3J1, Canada \\ Mahdi Sadjadi $\$$ \\ Department of Physics, Arizona State University, Tempe, AZ 85287-1504, USA \\ Brigitte Servatius 9 \\ Department of Mathematical Sciences, Worcester Polytechnic Institute, \\ 100 Institute Road, Worcester, MA 01609, USA \\ M. F. Thorpe ${ }^{* *}$ \\ Department of Physics, Arizona State University, Tempe, \\ AZ 85287-1504, USA and Rudolf Peierls Centre for Theoretical Physics, \\ University of Oxford, 1 Keble Rd, Oxford OX1 3NP, England
}

Finite pieces of locally isostatic networks have a large number of floppy modes because of missing constraints at the surface. Here we show that by imposing suitable boundary conditions at the surface, the network can be rendered effectively isostatic. We refer to these as anchored boundary conditions. An important example is formed by a two-dimensional network of corner sharing triangles, which is the focus of this paper. Another way of rendering such networks isostatic, is by adding an external wire along which all unpinned vertices can slide (sliding boundary conditions). This approach also allows for the incorporation of boundaries associated with internal holes and complex sample geometries, which are illustrated with examples. The recent synthesis of bilayers of vitreous silica has provided impetus for this work. Experimental results from the imaging of finite pieces at the atomic level needs such boundary conditions, if the observed structure is to be computer-refined so that the interior atoms have the perception of being in an infinite isostatic environment. 


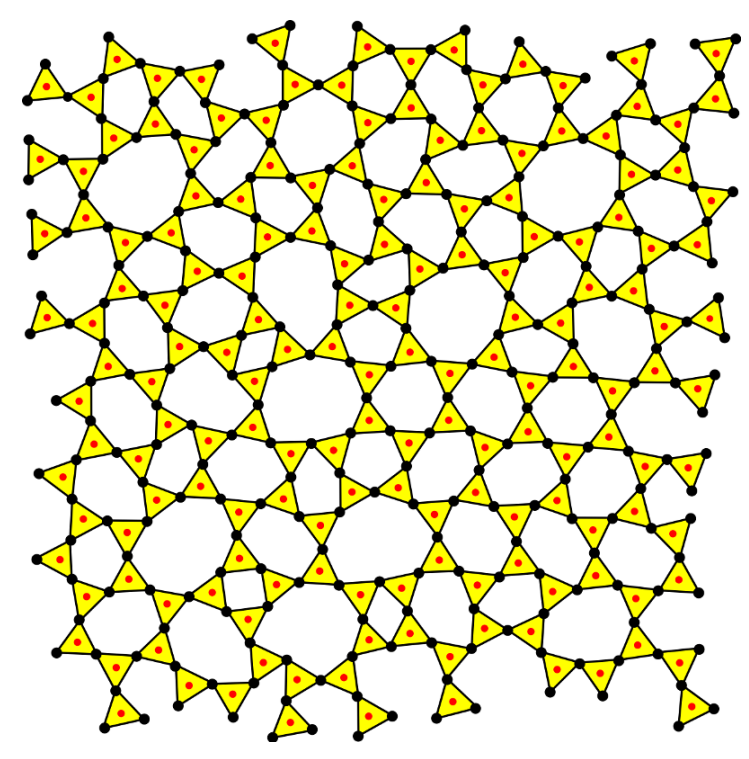

FIG. 1. Showing a piece of bilayer of vitreous silica imaged in SPM (Scanning Probe Microscope) ${ }^{5}$ to show the Si atoms as red discs and the $\mathrm{O}$ atoms as black discs. The local covalent bonding leads to the yellow almost-equilateral triangles that are freely jointed, which we will refer to as pinned. The triangles at the surface have either one or two vertices unpinned.

\section{INTRODUCTION}

Boundary conditions are paramount in many areas of computer modeling in science. At the atomic level, finite samples require appropriate boundary conditions in order that atoms in the interior behave as if they were part of a larger or infinite sample, or as closely to this as is possible. One example of this is the calculation of the electronic properties of covalent materials where the surface is terminated with $\mathrm{H}$ atoms so that all the chemical valency is satisfied. In this way the HOMO (highest occupied molecular orbital) and the LUMO (lowest unoccupied molecular orbital) states inside the sample can be obtained that are not very different from those expected in the bulk sample. In materials science the electronic band structure of a sample of crystalline $\mathrm{Si}$ could be obtained by determining the electronic properties of a finite cluster terminated with $\mathrm{H}$ bonds at the surface. In practice this is rarely done, as it is more convenient to use periodic boundary conditions and hence use Bloch's theorem, but this technique has been used recently in graphene nanoribbons $\sqrt{1}$.

For most samples, the nature of the boundary, fixed, free or periodic only alters the properties of the sample by the ratio of the number of atoms on the surface to those in the bulk. This ratio is $N^{-\frac{1}{d}}$ where $N$ is the number of atoms (later referred to as vertices) and $d$ is the dimension. Of course this ratio goes to zero in the thermodynamic limit as the size of the system $N \rightarrow \infty$ and leads to the important result that properties become independent of boundary conditions for large enough systems.

Similar statements can be made about the mechanical and vibrational properties of systems except for isostatic networks that lie on the border of mechanical instability. In this case the boundary conditions are important no matter how large $N$, and special care must be taken with devising boundary conditions so that the interior atoms behave as if they were part of an infinite sample, in as much as this is possible $e^{2 / 4}$.

In Figure 1, we show a part of a Scanning Probe Microscope (SPM) image ${ }^{5}$ of a bilayer of vitreous silica which has the chemical formula $\mathrm{SiO}_{2}$. The sample consists of an upper layer of tetrahedra with all the apexes pointing downwards where they join a mirror image in the lower layer. In the figure we show the triangular faces of the upper tetrahedra, which form rigid triangles with a (red) Si atom at the center and the (black) O atoms at the vertices of the triangles which are freely jointed to a good approximation. We refer to these networks as locally isostatic as the number of degrees of freedom of the equilateral triangle in two dimensions is exactly balanced by the shared pinning constraints ( 2 at each of the 3 vertices, so that $3-2 \times 3 / 2=0$ ). While the $3 \mathrm{D}$ bilayers are locally isostatic, so too are the $2 \mathrm{D}$ projections of corner-sharing triangles which are the focus of this paper. We will use the Berlin A sample as the example throughout ${ }^{6 / 7]}$ so that we can focus on this single geometry for pedagogical purposes.

In this paper, we show rigorously that there are various ways to add back the exact number of missing constraints at the surface, in a way that they are sufficiently uniformly distributed around the boundary that the network is guaranteed to be isostatic everywhere. There is some limited freedom in the precise way these boundary conditions are implemented, and the boundary can be general enough to include internal holes. The proof techniques used here 


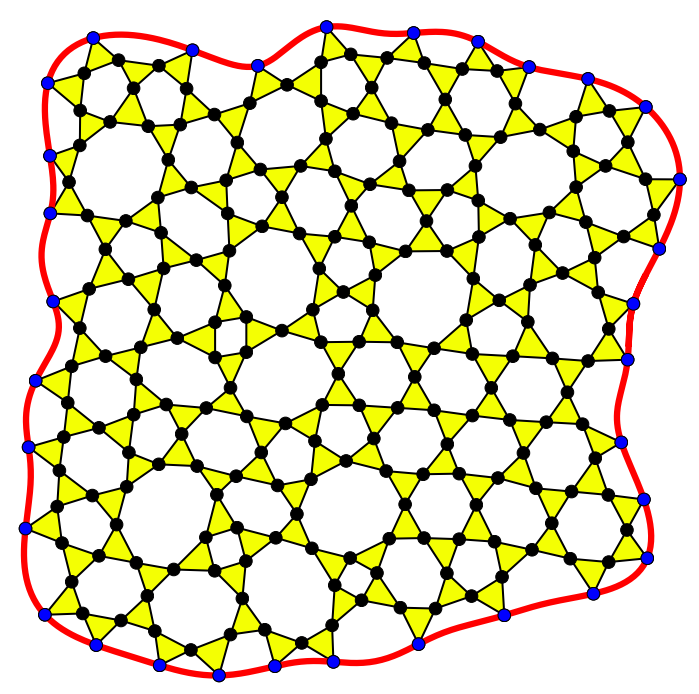

FIG. 2. Illustrating sliding boundary conditions, used for a piece of the sample shown in Figure 1 The boundary sites are shown as blue discs and the 3 purple triangles at the lower left Figure 3 have been removed. The red Si atoms at the centers of the triangles in Figure 1 have also been removed for clarity. The boundary is formed as a smooth analytic curve by using a Fourier series with 16 sine and 16 cosines terms to match the number of surface vertices, where the center for the radius $r(\theta)$ is placed at the centroid of the 32 boundary vertices 12 . Note that sliding boundary conditions do not require an even number of boundary sites.

involves showing that all subgraphs have insufficient edge density for redundancy to occur ${ }^{8}$. In the appendix, we give an algorithmic desctiption of our boundary conditions and discuss in detail how to ensure the resulting boundary is sufficiently generic.

Using the pebble game ${ }^{9110}$, we verified on a number of samples that anchored boundary conditions in which alternating free vertices are pinned results in a global isostatic state. The pebble game is an integer algorithm, based on Laman's theorem ${ }^{8}$, which for a particular network performs a rigid region decomposition, which involves finding the rigid regions, the hinges between them, and the number of floppy (zero-frequency) modes. We have used it to confirm that the locally isostatic samples such as that in this paper are isostatic overall with anchored boundary conditions. The results of this paper imply that, under a relatively mild connectivity hypothesis, this procedure is provably correct, and thus, relatively robust. Additionally, the necessity of running the pebble game for each individual case is avoided.

Figure 2 shows sliding boundary condition 11 . These make use of a different, simpler kind of geometric constraint at each unpinned surface site. The global effect on the network's degrees of freedom is like that of the anchored boundary conditions, and this setup is computationally reasonable. At the same time, the proofs for this case are simpler, and generalize more easily to handle situations such as holes in the sample.

In Figure 3, we show the anchored boundary conditions. We have trimmed off the surface triangles in Figure 1 that are only pinned at one vertex. This makes for a more compact structure whose properties are more likely to mimic those of a larger sample, and makes our mathematical statements easier to formulate. In addition we have had to remove the 3 purple triangles at the lower right hand side in order to get an even number of unpinned surface sites. When the network is embedded in the plane, this is possible, except for very degenerate samples (see Figure 3 ).

\section{COMBINATORIAL ANCHORING}

Intuitively, the internal degrees of freedom of systems like the ones in Figures 1 and 3 correspond to the corners of trianges that are not shared. This is, in essence, the content of Lemma 1 proved below. Proving Lemma 1 requires ruling out the appearance of additional degrees of freedom that could arise from sub-structures that contain more constraints than degrees of freedom.

The essential idea behind combinatorial rigidity $\sqrt{13}$ is that generically all geometric constraints are visible from the topology of the structure, as typified by Laman's 8 striking result showing the sufficiency of Maxwell counting ${ }^{14}$ in dimension 2. Genericity means, roughly, that there is no special geometry present; in particular, generic instances of 


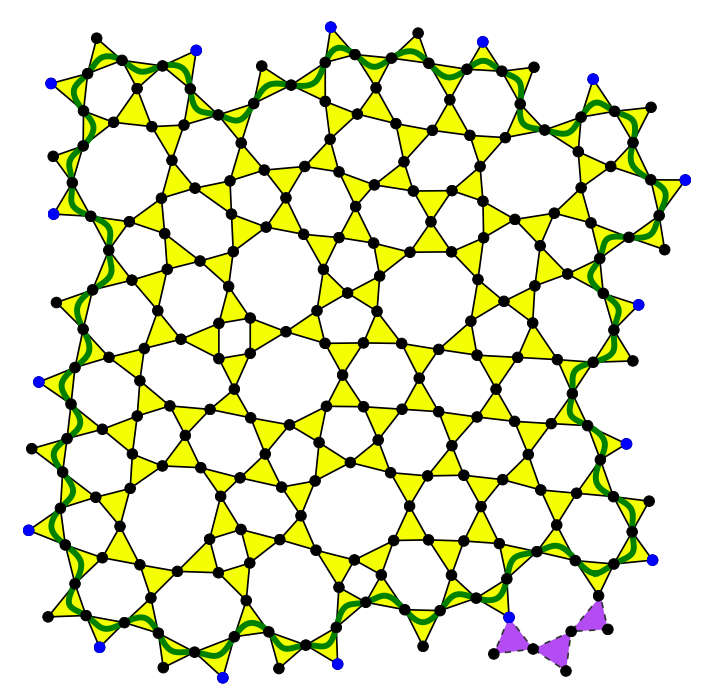

FIG. 3. Illustrating the anchored boundary conditions used for the sample shown in Figure 1 . The alternating anchored sites on the boundary are shown as blue discs and the 3 purple triangles at the lower right are removed to give an even number of unpinned surface sites. The red Si atoms at the centers of the triangles in Figure 1 have been suppressed for clarity.

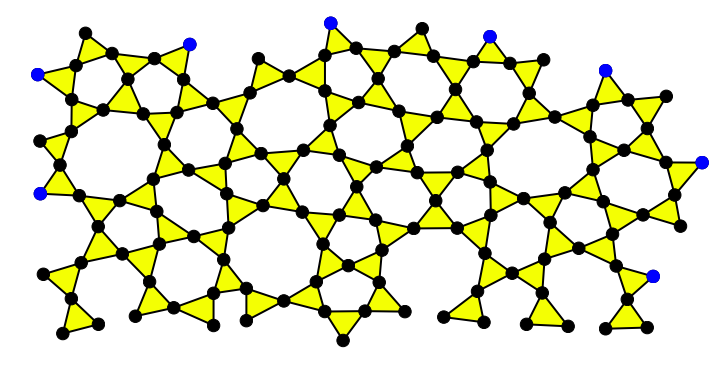

FIG. 4. Showing a typical subgraph from Figure 3 used in the proof that there are no rigid subgraphs larger than a single triangle. (See Lemma 9)

any topology are dense in the set of all instances.

In what follows, we will be assuming genericity, and then use results similar to Laman's, in that they are based on an appropriate variation of Maxwell counting. Our proofs have a graph-theoretic flavor, which relate certain hypotheses about connectivity $\sqrt{15}$ to hereditary Maxwell-type counts.

\section{A. Triangle ring networks}

We will model the flexibility in the upper layer of vitrious silica bilayers as systems of 2D triangles, pinned togehter at the corners. The joints at the corners are allowed to rotate freely. A triangle ring network is rigid if the only available motions preserving triangle shapes and the network's connectivity are rigid body motions; it is isostatic if it is rigid, but ceases to be so once any joint is removed. These are an examples of body-pin networks ${ }^{16}$ from rigidity theory.

The combinatorial model is a graph $G$ that has one vertex for each triangle and an edge between two triangles if they share a corner (Figure 6). Since we are assuming genericity, we will identify a geometric realization with the graph $G$ from now on. In what follows, we are interested in a particular class of graphs $G$, which we call triangle ring networks. The definition of a triangle ring is as follows: (a) $G$ has only vertices of degree 2 and 3; $G$ is 2-connected $\frac{17}{\text {; }}$; (b) there is a simple cycle $C$ in $G$ that contains all the degree 2 vertices, and there are at least 3 degree 2 vertices; (c) any edge cut set ${ }^{18}$ in $G$ that disconnects a subgraph containing only degree 3 vertices has size at least 3 .

To set up some terminology, we call the degree 2 vertices boundary vertices and the degrees 3 vertices interior vertices. A subgraph spanning only interior vertices is an interior subgraph. 

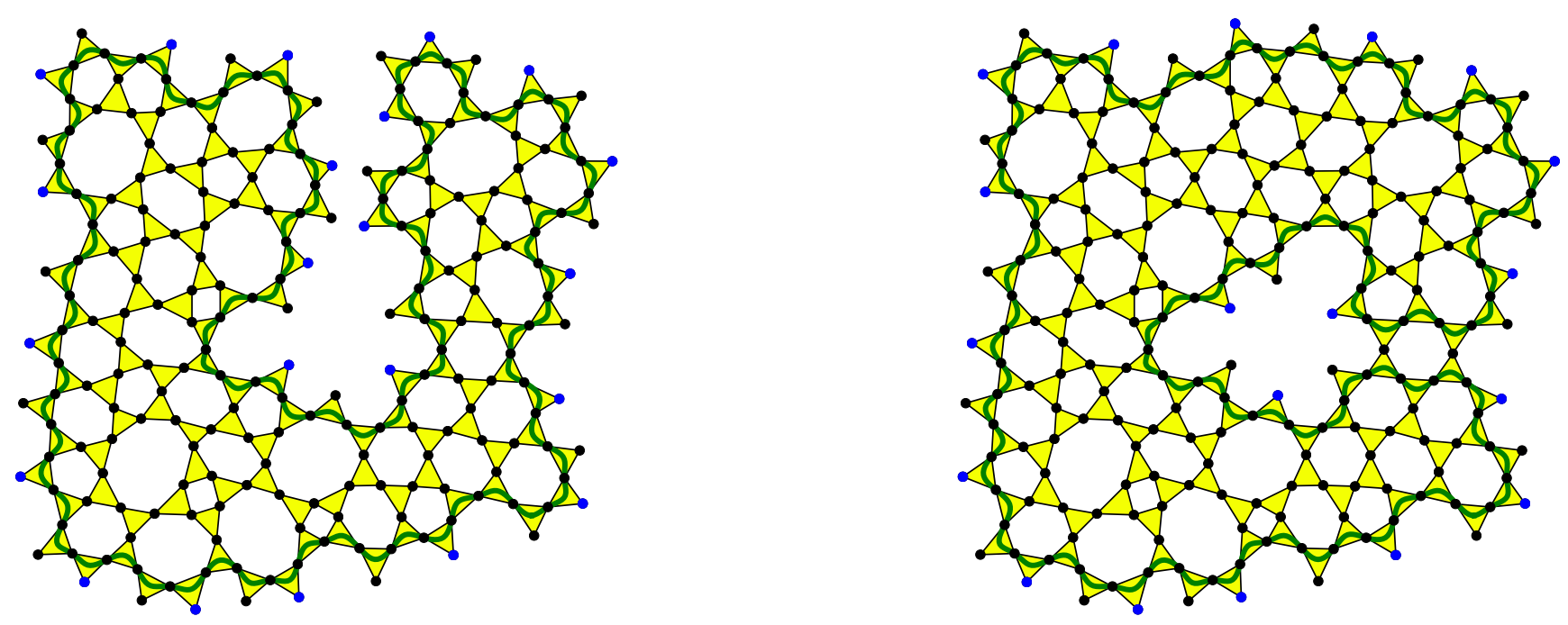

FIG. 5. Illustrating two, at first sight, more complex anchored boundary conditions that by our results can be used for the sample shown in Figure2, with the 3 purple triangles at the lower left are removed to give an even number of unpinned surface sites. The anchored sites are shown as blue discs, with an even number of surface sites in both graphs. The graph at the right has an even number of surface sites in both the outer and inner boundary. The red Si atoms at the centers of the triangles have been suppressed for clarity. The green line goes through the boundary triangles.

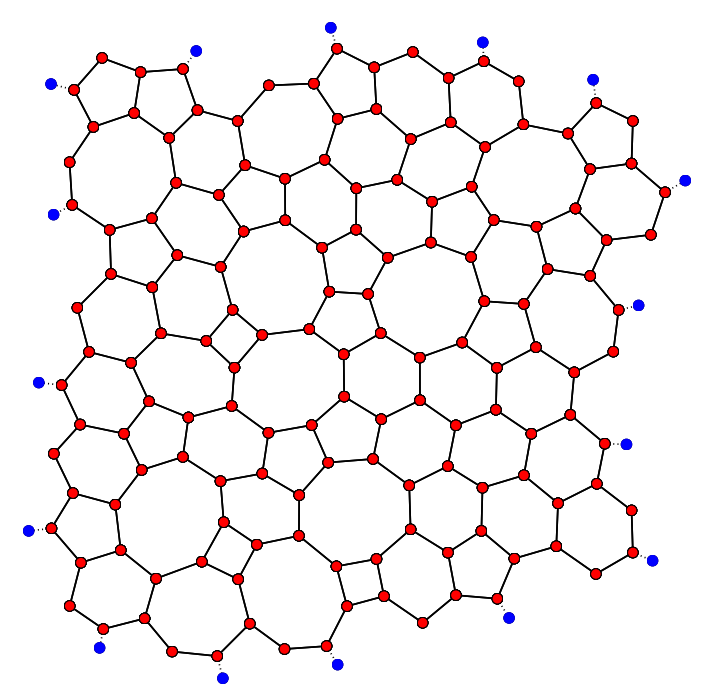

FIG. 6. The triangle ring network, complementary to that in Figure 3, where the Si atoms, shown as red discs, at the center of each triangle are emphasized in this three-coordinated network. Dashed edges are shown connecting to the anchored sites.

The reader will want to keep in mind the specific case in which $G$ is planar with a given topological embedding and $C$ is the outer face, as is the case in our figures. This means that subgraphs strictly interior to the outer face have only interior vertices, which explains our terminology. However, as we will discuss in detail later, the setup is very general. If the sample has holes, $C$ can leave the outer boundary and return to it: provided that it is simple, all the results here still apply.

A theorem of Tay-Whiteley $\frac{19120}{2}$ gives the degree of freedom counts for networks of 2-dimensional bodies pinned together. Generically, there are no stressed subgraphs in such a network, with graph $G$, of $v$ bodies and $e$ pins if and only if

$$
2 e^{\prime} \leq 3 v^{\prime}-3 \quad \text { for all subgraphs } G^{\prime} \subset G \text {. }
$$


where $v^{\prime}$ and $e^{\prime}$ are the number of vertices and edges of the subgraph. If (1) holds for all subgraphs, the rigid subgraphs are all isostatic, and they are the subgraphs where (1) holds with equality.

Lemma 1. Any triangle ring network $G$ satisfies (1).

Proof. Suppose the contrary. Then there is a vertex-induced subgraph $T$ on $v^{\prime}$ vertices that violates (1). If $T$ contains a vertex $v$ of degree 1 then $T-v$ also violates (1) so we may assume that $T$ has minimum degree 2 . In this case, $T$ has at most 2 vertices of degree 2 , since it has maximum degree 3 . In particular, $T$ may be disconnected from $G$ by removing at most 2 edges. If $T$ is an interior subgraph, we get a contradiction right away. Alternatively, at least one of the degree 2 vertices in $T$ is degree 2 in $G$, and so on $C$. If exactly one is, then $G$ is not 2-connected. If both are, then $T=G$ and there are only 2 boundary vertices. Either case is a contradiction.

Corollary 2. The rigid subgraphs of a triangle ring network $G$ are the subgraphs containing exactly 3 vertices of degree 2 and every other vertex has degree 3. Moreover, any proper rigid subgraph contains at most one boundary vertex of $G$.

Proof. The first statement is straightforward. The second follows from observing that if a rigid subgraph $T$ has two vertices on the boundary of $G$, then $G$ cannot be 2-connected, since all the edges detaching $T$ from $G$ are incident on a single vertex.

When $G$ is planar, these rigid subgraphs are regions cut out by cycles of length 3 in the Poincare dual. More generally in the planar case, subgraphs corresponding to regions that are smaller triangle ring networks with $t$ degree 2 vertices have $t$ degrees of freedom.

\section{B. Anchoring with sliders}

Now we can consider our first anchoring model, which uses slider pinning 11 . A slider constrains the motion of a point to remain on a fixed line, rigidly attached to the plane. When we talk about attaching sliders to a vertex of the graph, we choose a point on the corresponding triangle, and constrain its motion by the slider. In the results used below, this point should be chosen generically; for example the theory does not apply if the slider is attached at a pinned corner shared by two of the triangles. Since we are only attaching sliders to triangles corresponding to degree 2 vertices in $G$, we may always attach sliders at an unpinned triangle corner.

The notion of rigidity for networks of bodies with sliders is that of being pinned: the system is completely immobilized ${ }^{21}$ A network with sliders is pinned-isostatic if it is pinned, but ceases to be so if any pin or slider is removed.

The equivalent of the White-Whiteley counts in the presence of sliders is a theorem of Katoh and Tanigawa ${ }^{22}$, which says that a generic slider-pinned body-pin network $G$ is independent if and only if the body-pin graph satisfies (1) and

$$
2 e^{\prime}+s^{\prime} \leq 3 v^{\prime} \quad \text { for all subgraphs } G^{\prime} \subset G,
$$

where $s^{\prime}$ is the number of sliders on vertices of $G^{\prime}$. Here is our first anchoring procedure.

Theorem 3. Adding one slider to each degree 2 boundary vertex of a triangle ring network $G$ gives a pinned-isostatic network.

Proof. Let $T$ be an arbitrary subgraph with $v^{\prime}$ vertices and $v^{\prime \prime}$ vertices of degree at most 2. That (1) holds is Lemma 1. The fact that the only vertices of $T$ which get a slider are vertices with degree 2 in $G$ implies that (2) is also satisfied, and, by construction $2 e+s=3 v$.

We may think of this anchoring as rigidly attaching a rigid wire to the plane then constraining the boundary vertices to move on it. Provided that the wire's path is smooth and sufficiently non-degenerate, this is equivalent, for analyzing infinitesimal motions, to putting the sliders in the direction of the tangent vector at each boundary vertex. See also Figure 2 . 


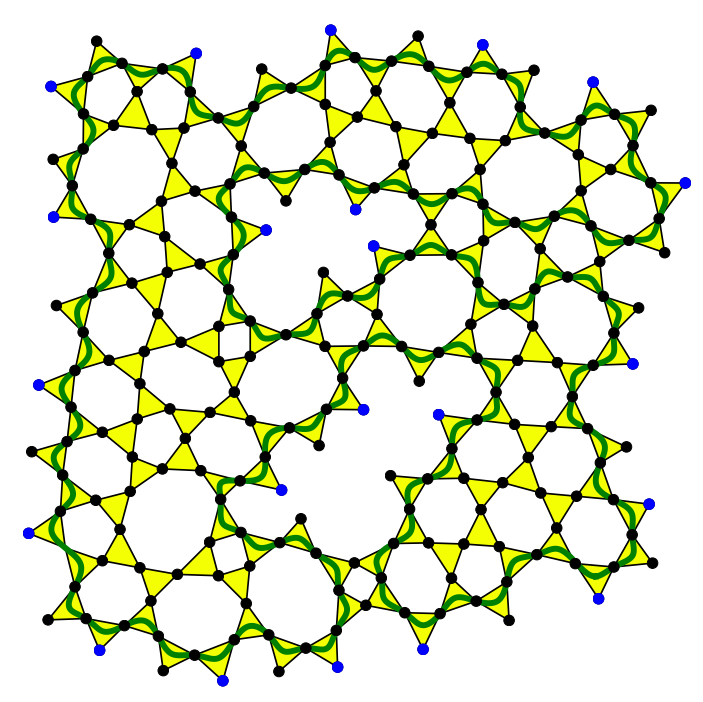

FIG. 7. Illustrating even more complex boundaries, developed from the sample shown in Figure 3 by removing triangles to form two internal holes. The boundary sites are shown as blue discs and the 3 purple triangles at the lower left Figure 3 have been removed. The red Si atoms at the centers of the triangles in Figure 1 have also been removed for clarity. The green line forms a continuous boundary which goes through all the surface sites which must be an even number. The anchored (blue) sites then alternate with the unpinned sites on the green boundary curve which has to cross the bulk sample in two places to reach the two internal holes. Here there are 32 boundary sites, 5 boundary sites in the upper hole and 7 in the lower hole, giving a total even number of 44 boundary sites. Where these crossings take place is arbitrary, but it is important that the anchored and unpinned surface sites alternate along whatever (green) boundary line is drawn.

\section{Anchoring with immobilized triangle corners}

Next, we consider anchoring $G$ by immobilizing (pinning) some points completely. Combinatorially, we model pinning a triangle's corner by adding two sliders through it. Since we are still using sliders, the definitions of pinned and pinned-isostatic are the same as in the previous section.

The analogue for 2 when we add sliders in groups of 2 is:

$$
2 e^{\prime}+2 s^{\prime} \leq 3 v^{\prime} \quad \text { for all subgraphs } G^{\prime} \subset G,
$$

where $s^{\prime}$ is the number of immobilized corners.

Theorem 4. Let $G$ be a triangle ring network with an even number $t$ of degree 2 vertices on $C$. Then, following $C$ in cyclic order, pinning every other boundary vertex that is encountered results in a pinned-isostatic network.

Proof. Let $T$ be an arbitrary subgraph of $G$. If at most one of the vertices of $T$ are pinned, there is nothing to do. For the moment, suppose that no vertex of degree 1 in $T$ is pinned. Let $t$ be the number of pinned vertices in $T$.

We will show that for each of the $t$ pinned vertices, there is a distinct unpinned vertex of degree 1 or 2 in $t$. This implies that $2 e^{\prime} \leq 3 v^{\prime}-2 t$ in $T$, at which point we know (3) holds for $T$.

To prove the claim, let $v$ be a pinned vertex of $T$. Traverse the boundary cycle $C$ from $v$. Let $w$ be the next pinned vertex of $T$ that is encountered. If the chain from $v$ to $w$ along $C$ is in $T$, the alternating pattern provides an unpinned degree 2 vertex that is degree 2 in $G$. Otherwise, this path leaves $T$, which can only happen at a vertex with degree 1 or 2 in $T$. Continuing the process until we return to $v$, produces at least $t$ distinct unpinned degree 2 vertices, since each step considers a disjoint set of vertices of $C$.

Now assume that $T$ does have a pinned vertex $v$ of degree 1 . The theorem will follow if (3) holds strictly for $T-v$. Let $w$ and $x$ be the pinned vertices in $T$ immediately preceding and following $v$. The argument above shows that there are at least 2 unpinned degree 1 or 2 vertices in $T$ on the path in $C$ between $w$ and $x$ on $C$. Since these are in $T-v$, we are done.

When there are an odd number of boundary vertices in $G$, Theorem 4 does not apply. This next lemma gives a simple reduction in many cases of interest. 

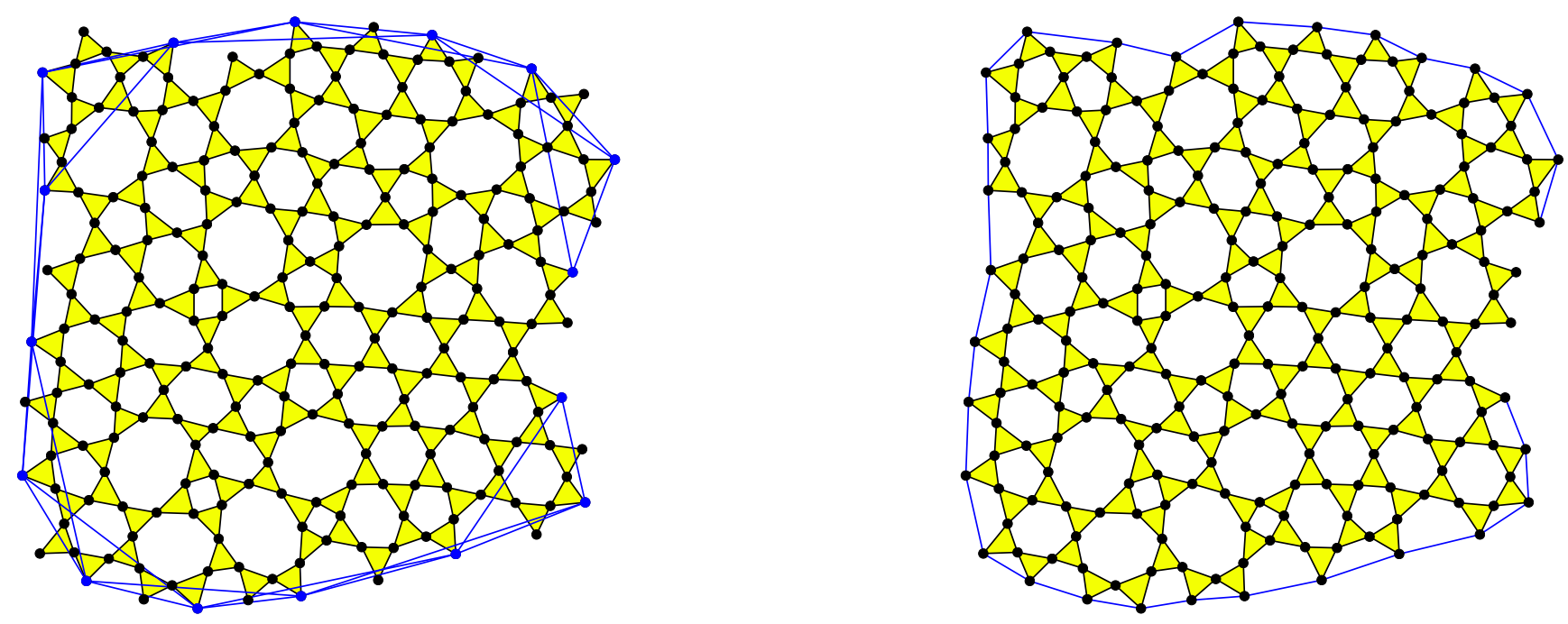

FIG. 8. Illustrating two additional boundary conditions used for the sample shown in Figure 3 , with the 3 purple triangles at the lower left removed to give an even number of unpinned surface sites. On the left, alternating surface sites are connected to one another through triangulation of first and second neighbors, with the last three connections not needed (these would lead to redundancy). Hence there are three additional macroscopic motions when compared to Figure 3 which can be considered as being pinned to the page rather than to the internal frame shown by green straight lines here. On the right we illustrate anchoring with additional bars which connect all unpinned surface sites, except again three are absent, to avoid redundancy, and to give the three additional macroscopic motions when compared to Figure 3

Theorem 5. Let $G$ be a planar triangle ring network, with $C$ the outer face. Suppose that there are an odd number $t$ of boundary vertices. If $G$ is not a single cycle, then it is possible to obtain a network with an even number of boundary vertices by removing the intersection of a facial cycle of $G$ with $C$, unless $G=C$.

Proof sketch. The connectivity requirements for a triangle ring network, combined with planarity of $G$ imply that the intersection of $C$ and any facial cycle $D$ of $G$ is a single chain. Every boundary vertex is in the interior of such a chain, so some facial cycle $D$ contributes an odd number of boundary vertices. Removing the edges in $D \cap C$ changes the parity of the number of boundary vertices.

\section{Anchoring with additional bars}

So far, we have worked with networks of triangles pinned together. Now we augment the model to also include bars between pairs of the triangles. We will always take the endpoints of the bars to be free corners of triangles that are boundary vertices in the underlying network $G$. Combinatorially we model this by a graph $H$ on the same vertex set as $G$, with an edge for each bar between a pair of bodies. In this case, the Tay-Whiteley count becomes:

$$
2 e^{\prime}+b^{\prime} \leq 3 v^{\prime}-3 \quad \text { for all subgraphs } G^{\prime} \subset G \text {. }
$$

where $e^{\prime}$ is the number of edges in $G^{\prime}$ and $b^{\prime}$ is the number of edges in $H$ spanned by the vertices of $G^{\prime}$. The anchoring procedures with sliders or immobilized vertices have analogues in terms of adding bars to create an isostatic network. These boundary conditions are illustrated on the right hand side of Figure 8. Also shown in Figure 8 in the left panel is a triangular scheme involving alternating unpinned surface sites, that is equivalent to anchoring. In both cases shown here the sample is free to rotate with respect to the page.

Theorem 6. If $G$ has boundary vertices $v_{1}, \ldots, v_{t}$, we obtain an isostatic framework by taking the edges of $H$ to be $v_{1} v_{2}, v_{2} v_{3}, \ldots, v_{t-3} v_{t-2}$.

Proof. Consider the $t-3$ new bars. By construction and Lemma 1 we have $2 e+t-3=3 v-t+t-3=3 v-3$. Corollary 2 and the connectivity hypotheses imply that no rigid subgraph of $G$ has more than 1 of its 3 degree 2 vertices on the boundary of $G$. This shows that no rigid subgraph of $G$ has a bar added to it.

Theorem 7. If $G$ has $t$ boundary vertices and $t$ is even, then taking $H$ to be any isostatic bar-joint network with vertex set consisting of $t / 2$ boundary vertices chosen in an alternating pattern around $C$ results in an isostatic network. 
A triangulated $t / 2$-gon is a simple choice for $H$.

Sketch. By Lemma 1, we are adding enough bars to remove all the internal degrees of freedom. The desired statement then follows from Theorem 4 by observing that pinning down the boundary vertices is equivalent, geometrically, to pinning down $H$ and then identifying the boundary vertices of $G$ to the vertices of $H$.

A result of White and Whiteley 23 on "tie downs", then gives:

Corollary 8. In the situation of Theorems 6 and 7 , adding any 3 sliders results in a pinned-isostatic network.

\section{E. Stressed regions}

The results so far have shown how to render a floppy triangle ring network isostatic or pinned-isostatic. It is interesting to know when adding a single extra bar or slider results in a network that is stressed over all its members. This is a somewhat subtle question when adding bars or immobilizing vertices, but it has a simple answer for the sliding boundary conditions.

We say that a triangle ring network is irreducible if: (a) every minimal 2 edge cut set either detaches a single vertex from $G$ or both remaining components contain more than one boundary vertex of $G$; (b) every minimal 3 edge cut set disconnects one vertex from $G$.

Lemma 9. A triangle ring network $G$ has no proper rigid subgraphs if and only if $G$ is irreducible.

Proof. Recall, from Corollary 2 , that a proper rigid subgraph $T$ of $G$ has exactly 3 vertices of degree 2 and the rest degree 3. Thus, $T$ can be disconnected from $G$ by a cut set of size 2 or 3 .

In the former case, Corollary 2 implies that exactly one of the degree 2 vertices in $T$ is a boundary vertex of $G$. This means that $T$ witnesses the failure of (a), and $G$ is not irreducible. Conversely, (a) implies that, for a 2 edge cut set not disconnecting one vertex, either side is either a chain of boundary vertices or has at least 4 vertices of degree 2.

Finally, observe that cut sets of size 3 are minimal if and only if they disconnect an interior subgraph on one side. Corollary 2 then implies that there is a proper rigid component that is an interior subgraph of $G$ if and only if (b) fails.

Theorem 10. Let $G$ be a triangle ring network anchored using the procedure of Theorem 3. Adding any bar or slider to $G$ results in a network with all its members stressed if and only if $G$ is irreducible.

Proof. First consider adding a slider. Because $G$ is pinned-isostatic, the slider creates a unique stressed subgraph $T$. A result of Streinu-Theran ${ }^{11}$ implies that $T$ must have been fully pinned in $G$. Since any proper subgraph has an unpinned vertex of degree 1 or 2, 22 holds strictly. Thus, the stressed graph is all of $G \cdot 24$

If we add a bar, there is also a unique stressed subgraph. This will be all of $G$ by 11 , unless both endpoints of the bar are in a common rigid subgraph. That was ruled out by assuming that $G$ is irreducible.

\section{CONCLUSIONS}

In this paper we have demonstrated boundary conditions for locally isostatic networks that incorporate the right number of constraints at the surface so that the whole network is isostatic. These boundary conditions should be useful in numerical simulations which involve finite pieces of locally isostatic networks. The boundary can be quite complex and involve both an external boundary with internal holes.

Although our definition of a triangle ring network is most easily visualized when $G$ is planar and $C$ is the outer face, the combinatorial setup is quite a bit more general. The natural setting for networks with holes is to assume planarity, and then that all the degree 2 vertices are on disjoint facial cycles in $G$. The key thing to note is that the cycle $C$ in our definition does not need to be facial for Theorem 4. For example, in Figure 7, $C$ goes around the boundary of an interior face that contains degree 2 vertices. In general, the existence of an appropriate cycle $C$ is a non-trivial question, as indicated by Figure 7 (See also Figure 5 for other examples of complex anchored boundary conditions).

What is perhaps more striking is that Theorem 3 still applies whether or not such a $C$ exists, provided faces in $G$ defining the holes in the sample are disjoint from the boundary and each other.

In applying anchored boundary conditions, it is important that the complete boundary has an even number of unpinned sites, which can include internal holes, which must then be connected using the green lines shown in the various figures. This gives a practical way of setting up calculations with anchored boundary conditions in samples with complex geometries and missing areas. 


\section{ACKNOWLEDGMENTS}

Support by the Finnish Academy (AKA) Project COALESCE is acknowledged by LT. We thank Mark Wilson and Bryan Chen for many useful discussions and comments. This work was initiated at the AIM workshop on configuration spaces, and we thank AIM for its hospitality.

* Email: louis.theran@aalto.fi Web: http://theran.lt

$\dagger$ Email: a.nixon@lancaster.ac.uk; Web: http://www.lancaster.ac.uk/maths/about-us/people/anthony-nixon

¥ Email: elissa.ross@meshconsultants.ca Web: http://www.elissaross.ca

$\S$ Email: ssadjadi@asu.edu

ๆ Email: bservat@wpi.edu Web: http://users.wpi.edu/ bservat

** Email: mft@asu.edu/Web: http://thorpe2.la.asu.edu/thorpe

1 O. Hod, J. E. Peralta, and G. E. Scuseria, Phys. Rev. B 76, 233401 (2007)

2 M. Thorpe, Journal of Non-Crystalline Solids 182, 135 (1995)

3 T. C. Lubensky, C. L. Kane, X. Mao, A. Souslov, and K. Sun, Reports on Progress in Physics 78, 073901 (2015).

${ }^{4}$ W. G. Ellenbroek, V. F. Hagh, A. Kumar, M. F. Thorpe, and M. van Hecke, Phys. Rev. Lett. 114, 135501 (2015).

${ }^{5}$ L. Lichtenstein, C. Büchner, B. Yang, S. Shaikhutdinov, M. Heyde, M. Sierka, R. Włodarczyk, J. Sauer, and H.-J. Freund, Angewandte Chemie International Edition 51, 404 (2012)

6 M. Wilson, A. Kumar, D. Sherrington, and M. F. Thorpe, Phys. Rev. B 87, 214108 (2013)

7 A. Kumar, D. Sherrington, M. Wilson, and M. F. Thorpe, Journal of Physics: Condensed Matter 26, 395401 (2014)

8 G. Laman, J. Engrg. Math. 4, 331 (1970)

9 D. J. Jacobs and M. F. Thorpe, Phys. Rev. Lett. 75, 4051 (1995)

10 D. J. Jacobs and M. F. Thorpe, Phys. Rev. E 53, $3682(1996)$.

11 I. Streinu and L. Theran, Discrete Comput. Geom. 44, 812 (2010).

12 M. Sadjadi, M. Wilson, and M. F. Thorpe, "Computer refinement of experimentally determined structures at the atomic level," Preprint, in preparation (2015).

13 See, e.g., the monograph by Graver, et al ${ }^{25}$ for an introduction.

14 J. C. Maxwell, The London, Edinburgh, and Dublin Philosophical Magazine and Journal of Science 27, 294 (1864)

15 To make this paper somewhat self-contained, we will briefly explain the concepts we use. Our terminology is standard, and can be found in, e.g., the textbook by Bondy and Murty 26 .

16 Since only two triangles are pinned together at any point, we are dealing with the 2-dimensional specialization of body-hinge frameworks first studied by Tay ${ }^{20}$ and Whiteley ${ }^{19}$ in general dimensions. In $2 \mathrm{D}$, there is a richer combinatorial theory of "body-multipin" structures, introduced by Whiteley ${ }^{27}$. See Jackson and Jordán 28 and the references therein for an overview of the area.

17 This means that to disconnect $G$, we need to remove at least 2 vertices.

18 This is a inclusion-wise minimal set of edges that, when removed from $G$, results in a graph 2 connected components.

19 W. Whiteley, SIAM J. Discrete Math. 1, 237 (1988).

20 T.-S. Tay, Graphs Combin. 5, 245 (1989)

21 Rigid body motions are not "trivial", because slider constraints are not preserved by them.

22 N. Katoh and S.-i. Tanigawa, SIAM J. Discrete Math. 27, 155 (2013)

23 N. L. White and W. Whiteley, SIAM J. Algebraic Discrete Methods 4, 481 (1983).

${ }^{24}$ It is worth noting that, so far, irreducibility of $G$ was not required. It is needed only for adding bars.

25 J. Graver, B. Servatius, and H. Servatius, Combinatorial rigidity, Graduate Studies in Mathematics, Vol. 2 (American Mathematical Society, Providence, RI, 1993) pp. x+172.

26 J. A. Bondy and U. S. R. Murty, Graph theory, Graduate Texts in Mathematics, Vol. 244 (Springer, New York, 2008).

27 W. Whiteley, Discrete Comput. Geom. 4, 75 (1989)

28 B. Jackson and T. Jordán, Discrete \& Computational Geometry 40, 258 (2008)

29 M. de Berg, O. Cheong, M. van Kreveld, and M. Overmars, Computational geometry, 3rd ed. (Springer-Verlag, Berlin, 2008) pp. xii+386, algorithms and applications.

30 F. J. Király, R. Tomioka, and L. Theran, "The algebraic combinatorial approach for low-rank matrix completion," To appear in JMLR (2015).

31 For example, given as a doubly-connected edge list. See, e.g., Section 2.2 of the textbook by de Berg, et al. ${ }^{29}$.

32 See the appendix of Király et al ${ }^{[30}$ for a detailed justification of this and similar statements relating genericity and random sampling. 


\section{Appendix A: Implementation details}

In this appendix we give an algorithmic description of our boundary conditions, including how to ensure that the sliders are chosen generically. Before describing the algorithms, we give more detail on how to encode a triangle ring network and the associated set of first-order geometric constraints.

\section{Encodings}

Computationally, it is convenient to work not only with the body graph $G$, as in the main body of the paper, but also with its line graph $G^{*}$ that has as its vertices the triangle corners and edges the triangle sides. We denote by $n$ and $m$ the number of vertices and edges in $G$ and $n^{*}$ and $m^{*}$ the same quantities for $G^{*}$. Vertices in $G$ are denoted by $v, w, \ldots$ and vertices in $G^{*}$ by $v^{*}, w^{*}, \ldots$. We assume that there is a constant-time mapping $\tau: V(G) \rightarrow V\left(G^{*}\right)^{3}$ that maps each vertex $v$ of $G$ to the associated triangle $\left\{v_{v}^{*}, w_{v}^{*}, x_{v}^{*}\right\}$ in $G^{*}$. For each boundary vertex $v$ of $G, \tau(v)$ will have a unique degree 2 vertex, which we denote by $T(v)$.

Experimentally, $G^{*}$ will always be immediately visible. It is also computable in time $O(n)$ from $G$. If $G$ is planar with given facial structure ${ }^{31}$, then $G^{*}$ also has an natural planar embedding, and vice-versa. Further, if $G^{*}$ contains no pair of facial triangles with a common edge, then $G$ is determined by $G^{*}$. This is the case in all of our examples.

We also assume that we have access to the coordinates of the vertices of $G^{*}$. We denote these by $p\left(v^{*}\right)=\left(x_{v^{*}}, y_{v^{*}}\right)$ for each vertex $v^{*}$ of $G^{*}$ and call $p$ a placement.

\section{First-order geometric constraints}

The allowed first-order motions $\dot{p}$ of a triangle ring network $G$ satisfy the system

$$
\left\langle p\left(v^{*}\right)-p\left(w^{*}\right), \dot{p}\left(v^{*}\right)-\dot{p}\left(w^{*}\right)\right\rangle=0 \quad \text { for all edges } v^{*} w^{*} \in E\left(G^{*}\right) \text {. }
$$

We assume that $p$ maximizes the rank of A1, which happens for almost all choices of $p$. By the theorems in this paper, this rank is equal to $m^{*}$ when $G$ is a triangle ring network.

Now identify a set $S^{*} \subset V\left(G^{*}\right)$ of vertices to which we will add one slider constraint. Assign a vector $s\left(v^{*}\right)=$ $\left(a_{v^{*}}, b_{v^{*}}\right)$ to each $v^{*} \in S^{*}$. The slider constraints on the first-order motions are:

$$
\left\langle s\left(v^{*}\right), \dot{p}\left(v^{*}\right)\right\rangle=0 \quad \text { for all } v^{*} \in S^{*} .
$$

To guarantee that the combined system A1 - A2 achieves its maximum rank ( $2 n^{*}$ for our sliding boundary condition), it is sufficient to pick each $s\left(v^{*}\right)$ uniformly at random from the unit circle 32

\section{Implementing slider-pinning}

Algorithm 1 shows how to implement the sliding boundary condition of Theorem 3.

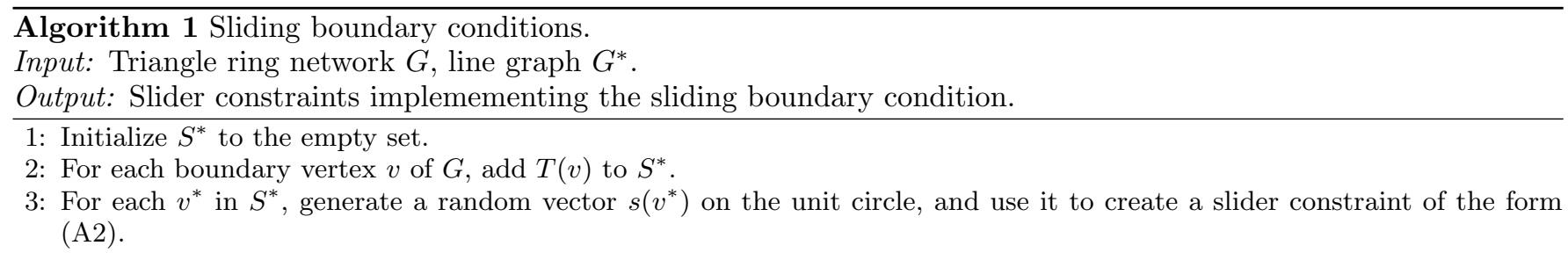

\section{Implementing immobilized vertices}

To implement Theorem 4, we could put two independent sliders at vertices of $G^{*}$. However, it is simpler to regard (A1) as a matrix and then discard the columns corresponding to immobilized vertices. 


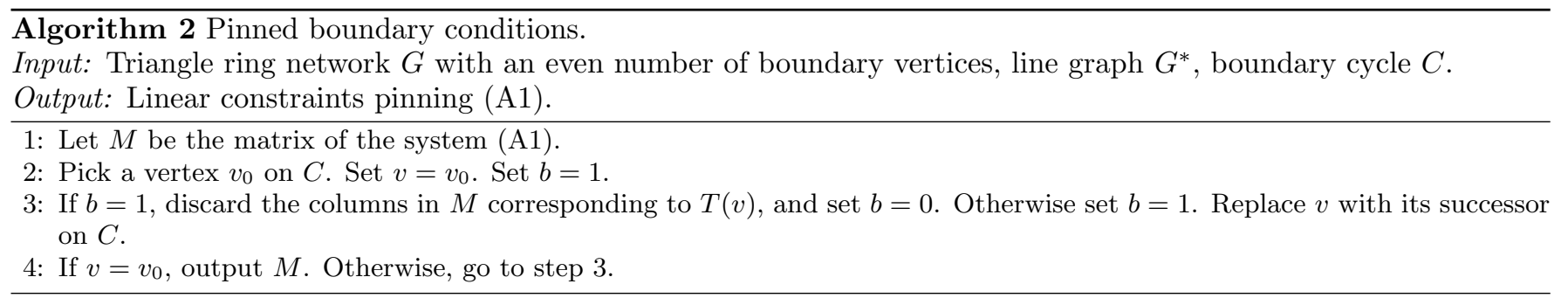

Observe that the loop implemented in steps 2-4 shows how to obtain the free corners of the boundary vertices of $G$.

\section{Implementing anchoring with additional bars}

Anchoring with additional bars amounts to adding edges to $G^{*}$. Thus, we describe them graph theoretically only. If the geometric constraints are desired, simply use the new graph to write down (A1).

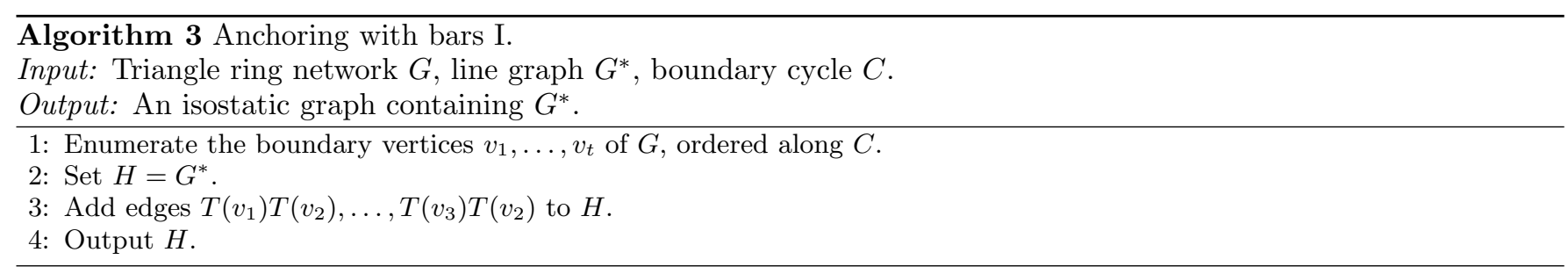

The left panel of Figure 8 takes the graph $H$ from Theorem 7 to be a "zig-zag triangulation" of a polygon, which is easily seen to be isostatic. This next algorithm gives the implementation of Theorem 7 using this choice.

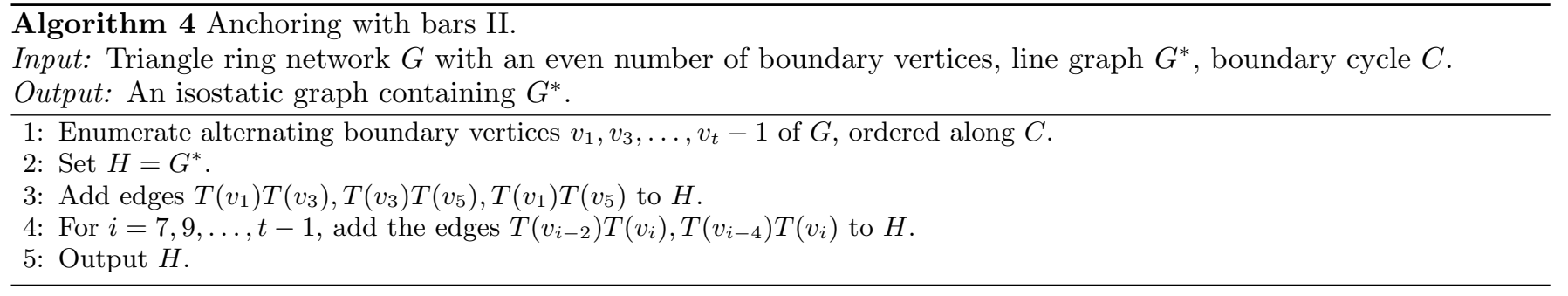

